Int. J. Dev. Biol. 50: 637-643 (2006)

doi: $10.1387 / \mathrm{ijdb} .052098 \mathrm{ah}$

Original Article

\title{
Formation of a successional dental lamina in the zebrafish (Danio rerio): support for a local control of replacement tooth initiation
}

\author{
ANN HUYSSEUNE* \\ Biology Department, Ghent University, Belgium
}

\begin{abstract}
In order to test whether the formation of a replacement tooth bud in a continuously replacing dentition is linked to the functional state of the tooth predecessor, I examined the timing of development of replacement teeth with respect to their functional predecessors in the pharyngeal dentition of the zebrafish. Observations based on serial semithin sections of ten specimens, ranging in age from four week old juveniles to adults, indicate that (i) a replacement tooth germ develops at the distal end of an epithelial structure, called the successional dental lamina, budding off from the crypt epithelium surrounding the erupted part of a functional tooth; (ii) there appears to be a developmental link between the eruption of a tooth and the formation of a successional dental lamina and (iii) there can be a time difference between successional lamina formation and initiation of the new tooth germ, i.e., the successional dental lamina can remain quiescent for some time. The data suggest that the formation of a successional lamina and the differentiation of a replacement tooth germ from this lamina, are two distinct phases of a process and possibly under a different control. The strong spatio-temporal coincidence of eruption of a tooth and development of a successional dental lamina is seen as evidence for a local control over tooth replacement.
\end{abstract}

KEY WORDS: tooth replacement, epithelial stem cells, zebrafish, Danio rerio

\section{Introduction}

Most tooth-bearing vertebrates (the notable exception being mammals) replace their teeth throughout life. In teleost fish, patterns of adjacent or alternate tooth initiation, set up by the firstgeneration teeth, can easily turn into regular replacement patterns where new teeth are initiated simultaneously every second, or even every third position. We have suggested that, once established, the replacement pattern appears to be maintained, as a kind of "default" state (Huysseune and Witten, 2006). Variations and modifications in this pattern are nevertheless common and suggest that tooth replacement is under local control. A regular, albeit variable pattern of tooth replacement is also present in the zebrafish (Van der heyden et al., 2001; Huysseune and Witten, 2006), a model which is increasingly used for studies on tooth patterning and replacement. In zebrafish, the teeth are nevertheless restricted to the pharyngeal region (cf. Huysseune et al., 1998), which represents a derived state compared to basal osteichthyans. The plesiomorphic condition for osteichthyans is one where teeth are distributed throughout the oral and pharyngeal cavity; more derived taxa have their dentition restricted to marginal, palatal and pharyngeal teeth. The cypriniforms, to which the zebrafish belongs, represent an even further derived state, where oral teeth have been lost as well, leaving only pharyngeal teeth.

Earlier, we have suggested that the formation of a replacement tooth in zebrafish could be triggered when a fully differentiated tooth erupts, thereby activating putative stem cells (Huysseune and Thesleff, 2004). If this link between eruption and production of a new tooth bud in the zebrafish is valid, then all erupted teeth should be associated with a tooth germ that is at least in an initiation phase. Along the same line of thought, one can speculate that there is a correlation between the state of differentiation of the replacement tooth germ and the state of functionality (i.e., an estimation of the time interval the tooth has been functional) of the erupted predecessor.

An earlier study on tooth replacement in juvenile and adult

Abbreviations used in this paper: ab, attachment bone; dl, dental lamina; $\mathrm{ft}$, functional tooth; rt, replacement tooth; tb, tooth base.

*Address correspondence to: Prof. Dr. Ann Huysseune. Biology Department, Ghent University, K.L. Ledeganckstraat 35, 9000 Gent, Belgium.
Fax +32-9-264-5344. e-mail: Ann.Huysseune@UGent.be 
zebrafish (Van der heyden et al., 2001) revealed that not all functional teeth were associated with a replacement tooth. However, these observations were made on cleared and stained material, which reveals tooth germs only from a moment at which mineralisation has started, i.e. in an advanced state of differentiation (in the above study, also unstained enameloid caps were considered). Germs in a stage of initiation, morphogenesis, or early cytodifferentiation can only be traced on histological sections.

In the present study, I used serial semithin sections through the dentition of zebrafish ranging in age from four week old juveniles to adults ( 9 to $32 \mathrm{~mm} \mathrm{SL}$ ) to examine (1) whether every erupted tooth is associated with a replacement tooth germ and (2) whether there is a correlation between the state of functionality of a tooth and of its successor.

\section{Results}

\section{Histological characteristics of the different categories of functional and replacement teeth}

Erupted teeth could be assigned to one of the following distinctive categories: attaching, young, mature, old, or in resorption (Fig. 1). Teeth in the process of attachment have a very cellular pulp cavity, tall, columnar and polarized odontoblasts in the tip of the pulp cavity, somewhat plumper, but active odontoblasts along the walls of the pulp cavity and polarized and active osteoblasts at the level of the attachment bone, which is still thin (Fig. 2A). Despite the little amount of attachment bone matrix, such teeth are erupted, i.e., have their tip exposed in the pharyngeal cavity. In young functional teeth, tall, polarized odontoblasts are still present in the tip of the pulp cavity. The latter is still quite cellular but less than in teeth in the process of attachment. Mature functional teeth have a much more empty-looking pulp cavity, filled with a loose network of stellate cells. At the tip of the pulp cavity, polarized cells nevertheless subsist (Fig. 2B). In old functional teeth, the pulp cavity is lined by flattened cells, if any. The pulp cavity gives a very loose and quite empty appearance. Often, the dentine
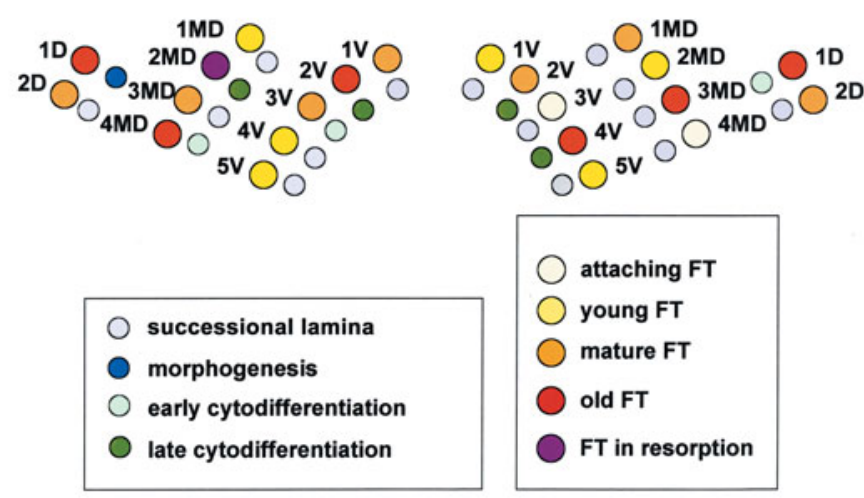

Fig. 1. Schematic representation of the pharyngeal dentition of a 11 $\mathbf{m m}$ SL zebrafish. The dentition was scored for the state of functionality of the erupted teeth (FT) (shades of yellow to red) and state of differentiation of the replacement teeth (grey, blue and shades of green). $1 \mathrm{~V}-5 \mathrm{~V}$ : 5 loci of the ventral (V) tooth row; 1MD-4MD: 4 loci of the mediodorsal (MD) tooth row; 1D-2D: 2 loci of the dorsal (D) tooth row.
TABLE 1

RELATIONSHIP BETWEEN THE STATE OF FUNCTIONALITY OF THE ERUPTED TEETH (FT) AND STATE OF DIFFERENTIATION OF THE REPLACEMENT TEETH (RT)

\begin{tabular}{lcccccc}
$\begin{array}{l}\text { Functionality of } \\
\text { erupted tooth }\end{array}$ & $\begin{array}{c}\text { Stage of } \\
\text { associated RT }\end{array}$ & V & MD & D & Total & Percentage \\
\hline attaching FT & SL & 8 & 7 & 2 & 17 & 100.0 \\
attaching FT & M & 0 & 0 & 0 & 0 & 0.0 \\
attaching FT & ED & 0 & 0 & 0 & 0 & 0.0 \\
attaching FT & LD & 0 & 0 & 0 & 0 & 0.0 \\
young FT & SL & 17 & 11 & 2 & 30 & 66.7 \\
young FT & M & 6 & 1 & 0 & 7 & 15.6 \\
young FT & ED & 4 & 0 & 1 & 5 & 11.1 \\
young FT & LD & 2 & 0 & 0 & 2 & 4.4 \\
mature FT & SL & 5 & 9 & 3 & 17 & 28.3 \\
mature FT & M & 2 & 1 & 3 & 6 & 10.0 \\
mature FT & ED & 10 & 2 & 2 & 14 & 23.3 \\
mature FT & LD & 15 & 5 & 3 & 23 & 38.3 \\
old FT & SL & 0 & 11 & 2 & 13 & 28.9 \\
old FT & M & 2 & 0 & 3 & 5 & 11.1 \\
old FT & ED & 1 & 4 & 6 & 11 & 24.4 \\
old FT & LD & 7 & 5 & 4 & 16 & 35.6 \\
FT in resorption & SL & 0 & 0 & 0 & 0 & 0.0 \\
FT in resorption & M & 0 & 1 & 0 & 1 & 7.7 \\
FT in resorption & ED & 0 & 0 & 0 & 0 & 0.0 \\
FT in resorption & LD & 4 & 5 & 3 & 12 & 92.3 \\
FT absent & SL & 0 & 0 & 0 & 0 & 0.0 \\
FT absent & $\mathrm{M}$ & 0 & 0 & 0 & 0 & 0.0 \\
FT absent & ED & 1 & 1 & 0 & 2 & 7.7 \\
FT absent & LD & 8 & 12 & 4 & 24 & 92.3 \\
Total & & $\mathbf{9 2}$ & $\mathbf{7 5}$ & $\mathbf{3 8}$ & $\mathbf{2 0 5}$ & \\
\hline A & & & & & & \\
\hline
\end{tabular}

Abbreviations: D, dorsal tooth row; ED, early cytodifferentiation; FT, functional tooth; LD, late cytodifferentiation; M, morphogenesis; MD, mediodorsal tooth row; RT, replacement tooth; SL, successional lamina; $\mathrm{V}$, ventral row tooth.

shows a metachromatic line, indicative of a secondary deposition (cf. Huysseune et al., 1998). Finally, in the most advanced category, obvious signs of resorption are visible: an irregular surface of the matrix, with resorption lacunae and the distinct presence of osteoclasts (Fig. 2C) (cf. Witten et al., 2001).

Replacement teeth were assigned to one of the four following stages: successional lamina stage (Fig. 2 D,E), morphogenesis (Fig. 2F), early cytodifferentiation, late cytodifferentiation. Detailed observations revealed that tooth germs develop from an epithelial structure in the shape of an invagination stretching over approximately $60 \mu \mathrm{m}$ in antero-posterior direction and penetrating, in average, around $45 \mu \mathrm{m}$ into the underlying mesenchyme. This epithelial structure precedes all following stages and was called the successional dental lamina (or, briefly, the successional lamina). This epithelial structure buds off from the epithelial fold that surrounds the erupted part of a functional tooth (called crypt, cf. Huysseune and Sire, 2004), close to the crypt base and at the posterior side of the erupted tooth (Figs. 2D,E, 3). Thus, on transverse sections, given that functional teeth point backwards, this epithelial structure can only be observed together with the tip of the predecessor. During morphogenesis stage the distal end of the successional lamina invaginates and forms a two-layered, bell-shaped enamel organ (Fig. 2F); during early cytodifferentiation the ameloblasts and odontoblasts differentiate and during late cytodifferentiation enameloid and dentine are deposited (cf. Laurenti et al., 2004). However, given the considerable size of the replacement teeth and the progressive differentiation from tip to base of the tooth, I included teeth with a small amount of matrix (enameloid) into the early differentiation stage as defined by 
Laurenti et al. (2004) (who worked mainly on first-generation teeth).

\section{Relationship between state of functionality of the func- tional teeth and state of differentiation of the replacement teeth}

All functional teeth appeared to be associated either with a successional lamina, or with a replacement tooth developing from it (Table 1, Fig. 4). A successional lamina was not present when teeth were not erupted.

Erupted functional teeth that were still in the process of ankylosis (i.e., attaching functional teeth) were always associated with a successional lamina as such (i.e., a tooth bud was not yet forming at its distal end). Thus, of the 17 loci with attaching teeth (whether from the ventral, mediodorsal or dorsal row), all (100\%) showed the presence of a successional lamina (Table 1, Fig. 4).

Despite the fact that, percentage-wise, the number of young replacement teeth decreased and the number of more advanced replacement teeth increased with ever more mature functional teeth (Fig. 4) there was no 1:1 correspondence between the state of functionality of the functional tooth and the state of development of its successor.

Thus, well attached but still young functional teeth appeared to be associated almost exclusively with replacement teeth at an early stage of development: with a successional lamina in 2/ 3 of the cases and with morphogenesis or early cytodifferentiation stage in another approximately $25 \%$. In two cases (repre- senting $4.4 \%$ ) the replacement tooth was well advanced with a considerable amount of matrix deposited (late cytodifferentiation stage).

In contrast, older functional teeth (both the categories 'old' and 'in resorption') were not necessarily associated with replacement teeth in an advanced stage of development. E.g., in 13 cases, old functional teeth were associated with a successional lamina only; one functional tooth in resorption was associated with a morphogenesis stage successor.

Finally, where the functional tooth was absent, the locus housed a replacement tooth either in early ( 2 cases, $7.7 \%$ ) or, mostly, in late cytodifferentiation stage (24 cases, 92.3\%) (Table 1, Fig. 4).

When the data on the three smallest and the three largest specimens were pooled separately and compared, old functional teeth appeared to be associated with a successional lamina only more often in larger specimens than in smaller specimens (38.9\% versus $8.3 \%$, compare with the average of $28.9 \%$ for all specimens together).

\section{Discussion}

In contrast to the data gathered from cleared and stained material (Van der heyden et al., 2001), it is clear that every functional tooth in the zebrafish is associated ab initio with an epithelial downgrowth, which I have called a successional dental lamina (or, briefly, a successional lamina), the distal end of which will subsequently develop into a replacement tooth

Fig. 2. One $\mu \mathrm{m}$ thick sections through the pharyngeal dentition of a zebrafish of $13 \mathbf{~ m m ~ S L}$. Functional teeth are shown in (A-C), their associated successional lamina or replacement tooth developing from it in (D-F). In (D-F) a dashed line indicates the boundary between the crypt epithelium and the successional lamina $(D, E$, arrowheads) or the developing replacement tooth bud (see arrowhead in (F)). Note that in the latter, the dental lamina has been taken up into the expanding enamel organ of the tooth bud. (A) Tooth in position $4 \mathrm{~V}$ in a phase of at-
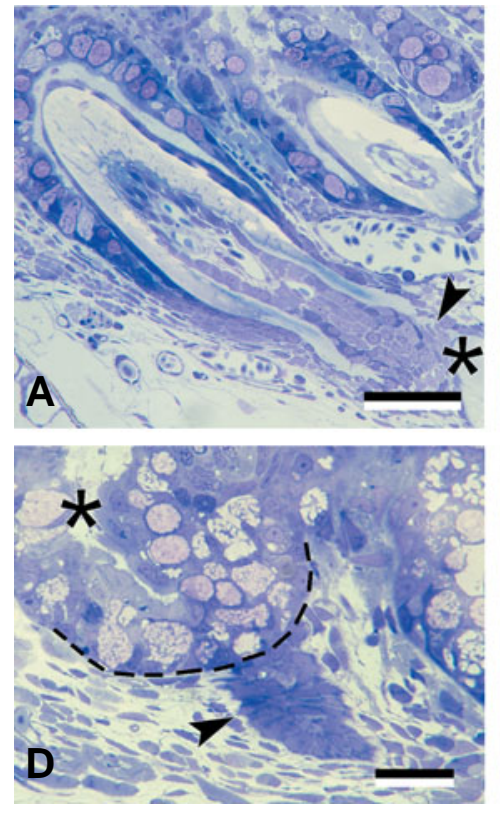
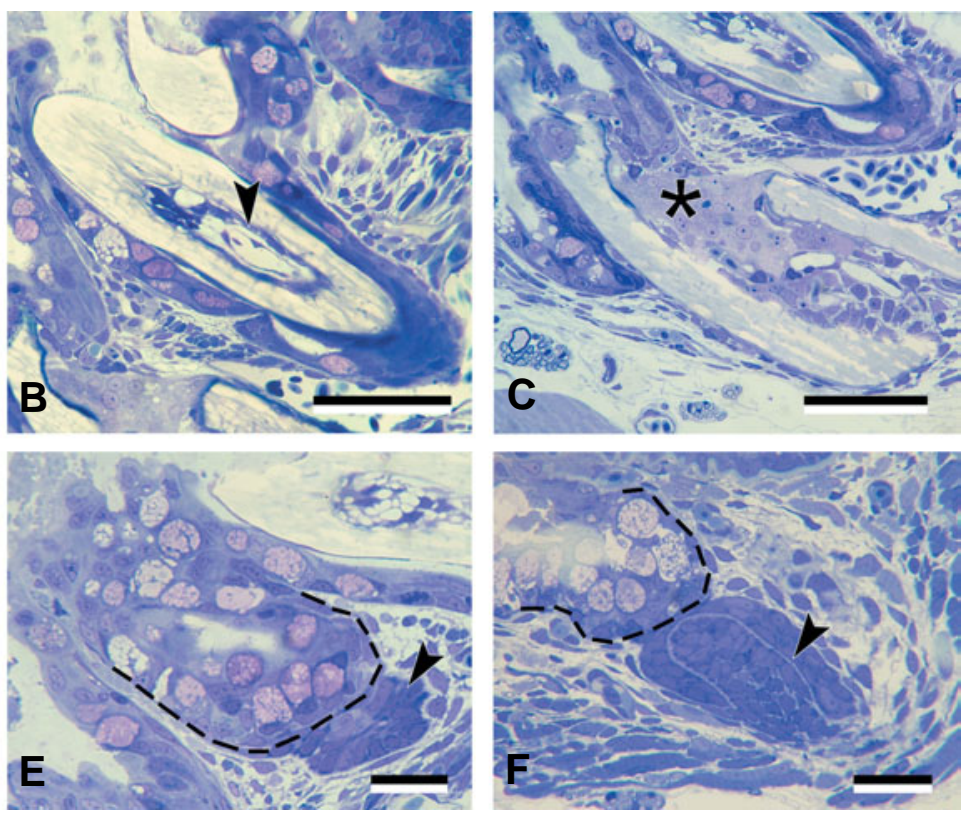

tachment (arrowhead) to the dentigerous bone (asterisk). Tall, polarized odontoblasts are found in the tip of the pulp cavity, more plump cells line the dentine surface. The tooth tip pierces the epithelium at another level of sectioning. Scale bar, $50 \mu m$. (B) Mature functional tooth in position $3 \mathrm{MD}$. Note the empty-looking pulp cavity and flattened cells lining the dentine (arrowhead). Scale bar, $50 \mu m$. (C) Functional tooth in resorption (position 4MD). Part of the tooth wall is broken away. The pulp cavity is largely filled with a multinucleated osteoclast (asterisk). Scale bar, 50 um. (D) Successional dental lamina (arrowhead) associated with the attaching functional tooth shown in (A). An asterisk marks the crypt surrounding the exposed part of the functional predecessor. Given that functional teeth in the animal point backwards and that the successional lamina buds off from the crypt surrounding the tip, only the tip of the predecessor can sometimes be observed at the same cross-sectional level as the successional lamina (not in D-F). Scale bar, $20 \mu \mathrm{m}$. (E) Successional lamina (arrowhead) associated with the mature functional tooth shown in (B). Scale bar, $20 \mu \mathrm{m}$. (F) Replacement tooth germ, stage of morphogenesis (arrowhead), associated with the functional tooth in resorption shown in (C). Scale bar, $20 \mu \mathrm{m}$. 
(Fig. 3). I also show that there can be a time difference between formation of the successional lamina and initiation of the tooth bud itself. Once the tooth bud starts to differentiate, the successional lamina is soon taken up into the enamel organ of the growing bud. This, together with the small size of the successional lamina and the fact that its tip usually goes on differentiating immediately into a tooth bud, is likely to make the successional lamina easily overlooked. The successional lamina was previously included in the early phases of morphogenesis by Van der heyden et al. (2000) because its possible temporary persistence as such remained unnoticed at the time. The successional lamina bears considerable resemblance to a dental lamina as described by Reif (1982) for actinopterygians: the successional lamina is discontinuous (i.e., there is a separate downgrowth for each tooth family) and it is also nonpermanent (i.e., it is formed anew for every replacement tooth). Importantly, the enamel organ of the first-generation teeth develops directly from the oral or pharyngeal epithelium, without the involvement of a lamina, not only in zebrafish (Huysseune et al., 1998; Laurenti et al., 2004), but also, e.g. in trout (Fraser et al., 2004) and in cichlids (Huysseune and Sire, 1997) and this may be a general feature for teleosts and non-mammalian tetrapods (cf. Huysseune and Sire, 1998; Sire et al., 2002).

Although the data suggest that together with an increase of functional life time of a predecessor, the developmental stage of its successor is, on average, ever more advanced, there is no strict $1: 1$ correspondence between the state of functionality of a tooth and the developmental stage of its successor. Such would only be the case if one would find that, e.g., all young functional teeth are associated with morphogenesis stage replacement teeth, all old functional teeth with late cytodifferentiation stage replacement teeth, etc. Rather, the data presented here reveal two important new findings regarding the mechanisms that control replacement tooth formation in zebrafish: (i)
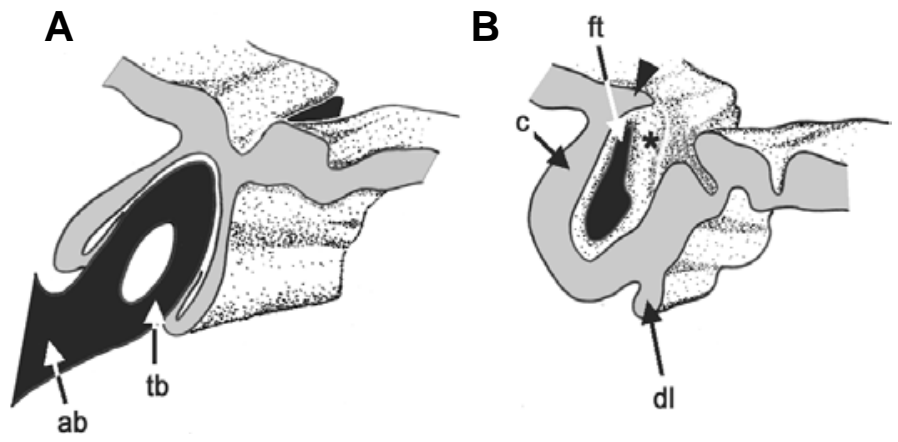

Fig. 3. Schematic representation of an erupted functional tooth and its associated successional dental lamina. Schematic representation, based on a 3D-reconstruction of tooth $1 \mathrm{~V}$ in a zebrafish of $11 \mathrm{~mm} \mathrm{SL}$, of the successional dental lamina (dl) and its relationship to the crypt (c) surrounding the tip of the erupted functional tooth $1 \mathrm{~V}(\mathrm{ft})$. The reconstruction is artificially divided in two parts to show two levels of sectioning of tooth $1 \mathrm{~V}$ : anteriorly $(\mathbf{A})$, where the tooth base $(t b)$ is continuous with the attachment bone (ab) and posteriorly (B), where only the tooth tip (ft) is left. Note that the tooth tip, despite lying freely in the crypt, is still hidden by a fold of the epithelial surface (arrowhead). The epithelial crypt closes behind this tooth tip (asterisk). Grey, sectioned epithelium; black, tooth $1 \mathrm{~V}$. there appears to be a developmental link between the eruption of a tooth and the development of a successional dental lamina (see below for a definition of tooth eruption in zebrafish) and (ii) the successional dental lamina can persist for a while as such, without giving rise at its distal end to a replacement tooth bud and this appears to be more often the case in larger (older) compared to smaller (younger) animals.

(i) The developmental link between tooth eruption and replacement tooth formation was already noticed in organotypic cultures: first-generation teeth which develop up to attachment stage fail to erupt and do not produce a replacement tooth germ (Van der heyden et al., 2005). Eruption of a tooth in zebrafish is not the result of tooth movement or of a final growth spurt of the tooth, but results from epithelial remodeling around the tip of the tooth (Huysseune and Sire, 2004). Through detachment of the epithelial cells covering the tooth tip and retraction of the epithelial layers, the tooth tip becomes exposed. This retraction displaces the epithelium, widens the crypt surrounding the exposed tooth tip and likely brings the epithelium into contact with a new mesenchymal environment. Possibly, such a newly established contact could activate the epithelium and lead to the production of a new downgrowth, irrespective of whether adult epithelial stem cells are involved (cf. Huysseune and Thesleff, 2004), progenitor cells of some sort, or even dedifferentiated epithelial cells. In this scenario, the developmental link between eruption and the formation of a successional lamina, as the anlage of the replacement tooth, would be a causal one. Alternatively, the two processes (eruption and formation of the successional lamina) may be two independent outcomes of the same (molecular) event. The search for candidate genes potentially involved in such an event can benefit from a comparison with hair formation. Despite being non-homologous features, hairs and teeth show many similarities in terms of being cycling structures, developing as a result of epithelial-mesenchymal interactions, etc. They can be considered an example of so-called generative homoplasy (Wake, 2003). Hairs and teeth are at least partly controlled by similar molecular cascades and many mutations affecting hairs also affect teeth (cf. Huysseune and Thesleff, 2004). In hairs, downregulation of Ecadherin through activation of Wnt signaling reduces cell adhesion in the stem cell niche, required to rearrange the epithelial cells to make a new bud (Barrandon, 2003; Jamora et al., 2003). Earlier, we have suggested that E-cadherin could also be involved in the remodeling of the epithelial layers covering the tooth tip prior to eruption (that is, inner and outer dental epithelium, possibly also a superficial pharyngeal epithelial cell layer), leading to cell detachment and exposure of the tooth tip (Huysseune and Sire, 2004; Huysseune and Thesleff, 2004). If $\mathrm{E}$-cadherin is at the same time downregulated in other parts of the enamel organ, notably the area of the outer dental epithelium from which the successional lamina will develop, this could lead to a loosening of cell contacts enabling the formation of a bud, similar to what has been suggested for hairs (Barrandon, 2003; Jamora et al., 2003). In this scenario, eruption and formation of the successional lamina would be independent outcomes of the same molecular event (such as downregulation of E-cadherin).

At this point, it is relevant to compare the situation in zebrafish with other vertebrates which also show continuous 
replacement. Both in amphibians (Wistuba et al., 2002; DavitBéal et al., pers. comm.) and in lizards (Edmund, 1969; Sire et al., 2002; Delgado et al., 2005) the successional lamina forms well before the predecessor has erupted. This makes a causal link between eruption and successional lamina formation unlikely but it does not preclude that successional lamina formation in zebrafish and tetrapods could be under a similar control. Like in zebrafish the successional lamina in amphibians and lizards is non-permanent (i.e. it disappears when the replacement tooth erupts) and discontinuous (i.e. there is a separate downgrowth for each replacement tooth). However, unlike in zebrafish, the successional lamina is quite long and therefore remains distinctive over a longer period of replacement tooth formation, before it is finally taken up into the growing enamel organ of the replacement tooth germ. The difference therefore seems to be quantitative, rather than qualitative. Several findings nevertheless caution for a careless extrapolation of zebrafish data to crownward osteichthyans (like tetrapods). Indeed, earlier studies on the molecular control of tooth formation in zebrafish have already revealed fundamental differences with that in mice (e.g. Laurenti et al., 2004; Jackman et al., 2004; Borday-Birraux et al., 2006).

(ii) Assuming that the state of functionality of a tooth (whether young, mature, old, or in resorption) reflects the time the tooth has been functional, the frequent presence of successional dental laminae associated with old functional teeth, with no indication of differentiation of their distal end into a tooth germ, suggests that the successional lamina can remain quiescent for some time. Some trigger, other than the state of the predecessor, appears to be responsible for starting the further development (i.e. the true initiation) of the replacement tooth in the zebrafish. In teleosts which display intramedullary (intraosseous) replacement (cf. Trapani, 2001), an often very long epithelial downgrowth develops from the crypt epithelium and penetrates the bone, before its distal end finally differentiates into a tooth germ within the medullary cavity of the tooth-bearing bone. This too suggests that a separate mechanism triggers the start of further differentiation of the tooth germ. The nature of this trigger is of high interest, given some remarkable parallels to the human dentition. In humans, a successional lamina appears on the lingual side of each enamel organ. In deciduous teeth, these lingual downgrowths give rise to the tooth germs of the permanent successors (incisors, canines, premolars) (Mjör and Fejerskov, 1979; Melfi, 1982; Bhaskar, 1990). Remarkably, such downgrowths are also said to be produced from the enamel organs of permanent teeth, but reported to eventually disappear (Berkovitz et al., 1992). Thus, like in zebrafish, there seems to be an uncoupling between the development of a successional dental lamina and the eventual proliferation of the cells at its distal end to give rise to a tooth germ. Moreover, in humans, the three permanent molars - which do not have deciduous predecessors - arise from a distal extension of the primary dental lamina in a period spread over several years. Mjör and Fejerskov (1979) correctly highlighted this discrepancy between the formation of the dental lamina and the formation of teeth developing from it, by stating "Although the overall activity of the dental lamina thus covers a considerable period of time (4-5 years) any particular portion of it only functions for a very short period before differentiation and desintegration." Recently, Wang et al. (2005) suggested that the transcription factor Runx2 might inhibit secondary tooth formation in mice by preventing the formation of an extension of the dental lamina for successional teeth. Humans affected from cleidocranial dysplasia (a disorder caused by the lack of expression of one allele of Runx2) have supernumerary teeth, that develop from secondary teeth and sometimes form an almost complete third dentition (Jensen and Kreiborg, 1990).

The intimate relationship which appears to exist between eruption of a tooth and formation of the successional lamina strongly suggests that there is a local control over replacement tooth formation, rather than a general type of control that orchestrates the formation of replacement teeth in the entire dentition. Such a general type of control, under the form of field substances, or morphogens, travelling over the jaw and eliciting replacement tooth formation in successive loci, has formerly been suggested to underlie the orderly and predictable

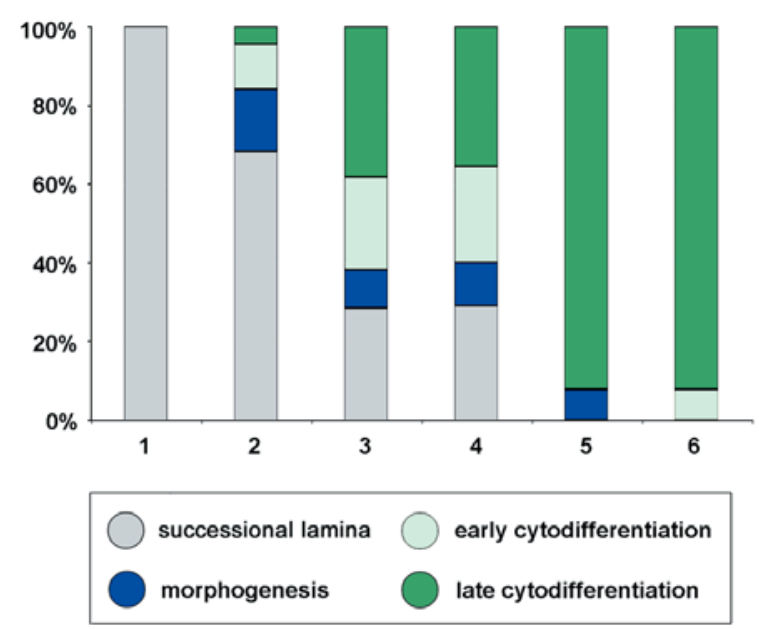

Fig. 4. Graphic representation of relationship between state of functionality of functional teeth and state of differentiation of their successor. Percentage of functional teeth 1 , attaching functional teeth $(F T)$; 2, young FT; 3, mature FT; 4, old FT; 5, FT in resorption) associated with specific stages of development of the replacement teeth; 6 , replacement teeth with predecessor absent. The data concern all 205 tooth positions examined.

patterns of tooth replacement often observed in non-mammalian vertebrates (see reviews by Berkovitz, 2000; Smith, 2003; Huysseune and Witten, 2006). Van der heyden et al. (2001) examined the order of replacement in the dentition of juvenile and adult zebrafish, based on around 90 cleared and stained specimens. Although one pattern emerged as the most common, these authors also showed the existence of considerable variation in this formula (up to $30 \%$ of the jaws examined). If replacement were to be under the control of some general field effect, one would not expect such a variation in the order of replacement.

Taken together, the detailed observations on serially sectioned zebrafish pharyngeal dentitions presented here suggest that there are two phases in the process of securing the formation of a replacement tooth, that are likely acting independently: the development of a successional lamina, linked to the 
eruption of the predecessor and the start of morphogenesis and differentiation of the tooth germ proper, which can, but not necessarily does, immediately follow the formation of the successional lamina. Epithelial stem cells, as a local control mechanism, could be involved in the first of these processes, but others need to be considered as well: a pool of pluripotent progenitor cells, dedifferentiation of epithelial cells, etc. The factor(s) triggering the further differentiation of the distal end of the successional lamina into a tooth germ remain to be elucidated. In particular, the question needs to be addressed whether the molecular cascade that controls this further differentiation is similar to that controlling initiation of first-generation teeth (cf. Laurenti et al., 2004; Jackman et al., 2004; Borday-Birraux et al., 2006) and to what extent principles of budding morphogenesis, involving interactions between activator and inhibitor substances, are applicable (cf. Peterková et al., 2000, 2002).

\section{Materials and Methods}

Zebrafish of $9,11,11.5,13,15,16.5,20,25$ and $32 \mathrm{~mm} \mathrm{SL}$ (a total of ten specimens) were anaesthetized using an overdose of MS222, fixed in a paraformaldehyde-glutaraldehyde solution, dehydrated and embedded in epon according to procedures described previously (cf. Huysseune and Sire, 1992). Serial, semithin (1 or $2 \mu \mathrm{m})$ transverse sections were prepared of the head and stained with toluidine blue.

First, the position (locus) of every functional tooth was identified by screening all sections in the pharyngeal region (i.e., positions $1 \mathrm{~V}$ to $5 \mathrm{~V}$ in the ventral tooth row, positions $1 \mathrm{MD}$ to $4 \mathrm{MD}$ in the mediodorsal row and $1 \mathrm{D}$ to $2 \mathrm{D}$ in the dorsal row, cf. Van der heyden and Huysseune, 2000). Next, for every position, the functional tooth and its successor were scored. Using histological characteristics, functional teeth (i.e., erupted and attached to the underlying bone) were assigned to one of the following categories: attaching, young, mature, old, or in resorption. Replacement teeth were assigned to one of the following stages: successional lamina stage, morphogenesis, early cytodifferentiation, late cytodifferentiation, the latter three according to features described previously (Laurenti et al., 2004) (for a definition of the successional lamina stage, see the results section) (Fig. 1). Since Van der heyden et al. (2001) showed that there is no relationship between the state of differentiation/ replacement of the right and the left halve of the dentition, both sides of each animal were scored separately. Thus, a total of 205 loci was scored, i.e. 15 less than the expected $220-11$ loci on each jaw - because of loss of some sections, or absence of functional teeth in some loci.

Finally, all observations for the three tooth rows were pooled and for each category of functional teeth percentages were calculated of the different stages of tooth germs associated with these teeth.

\section{Acknowledgements}

The author wishes to thank Mrs B. De Kegel, N. Van Damme, M. Soenens and $F$. Allizard for expert technical assistance in preparing the serial sections. Grants of the FWO $n^{\circ}$ G.0159.05 and BOF (Ghent University, grant $n^{\circ}$ 011V1203) are gratefully acknowledged.

\section{References}

BARRANDON, Y. (2003). Developmental biology - A hairy situation. Nature 422: 272-273.

BERKOVITZ, B.K.B. (2000). Tooth replacement patterns in non-mammalian vertebrates. In Development, Function and Evolution of Teeth. (Teaford, M., M. Smith \& M. Ferguson, Eds.), Cambridge University Press, Cambridge, pp. 186-200.

BERKOVITZ, B.K.B., HOLLAND, G.R. and MOXHAM, B.J. (1992). A Colour Atlas \& Textbook of Oral Anatomy. Second Edition. Wolfe Medical Publications Ltd, London.
BHASKAR, S.N. (1990). Orban's Oral Histology and Embryology. Eleventh Edition. Mosby Year Book, St. Louis.

BORDAY-BIRRAUX, V., VAN DER HEYDEN, C., DEBIAIS-THIBAUD, M., VERREIJDT, L., STOCK, D.W., HUYSSEUNE, A. and SIRE, J-Y. (2006). Expression of Dlx genes during the development of the zebrafish pharyngeal dentition: evolutionary implications. Evol. Dev. 8: 130-141.

DELGADO, S., DAVIT-BÉAL, T., ALLIZARD, F. and SIRE, J.-Y. (2005). Tooth development in a scincid lizard, Chalcides viridanus (Squamata), with particular attention to enamel formation. Cell Tiss. Res. 319: 71-89.

EDMUND, A.G. (1969). Dentition. In Biology of reptilia, vol I. (Gans, C., A.d'A. Bellairs and T.S. Parsons, Eds.), Academic Press, London, pp. 117-200.

FRASER, G.J., GRAHAM, A. and SMITH, M.M. (2004). Conserved deployment of genes during odontogenesis across osteichthyans. Proc. R. Soc. Lond. B271: 2311-2317.

HUYSSEUNE, A. and SIRE, J.-Y. (1992). Development of cartilage and bone tissues of the anterior part of the mandible in cichlid fish: a light and TEM study. Anat. Rec. 233: 357-375.

HUYSSEUNE, A. and SIRE, J.-Y. (1997). Structure and development of firstgeneration teeth in the cichlid Hemichromis bimaculatus (Teleostei, Cichlidae). Tissue Cel/29: 679-697.

HUYSSEUNE, A. and SIRE, J.-Y. (1998). Evolution of patterns and processes in teeth and tooth-related tissues in non-mammalian vertebrates. Eur. J. Oral Sci. 106 (Suppl. 1): 437-481.

HUYSSEUNE, A. and SIRE, J.-Y. (2004). The role of epithelial remodelling in tooth eruption in larval zebrafish. Cell Tiss. Res. 315: 85-95.

HUYSSEUNE, A. and THESLEFF, I. (2004). Continuous tooth replacement: the possible involvement of epithelial stem cells. BioEssays 26: 665-671.

HUYSSEUNE, A., VAN DER HEYDEN, C. and SIRE, J.-Y. (1998). Early development of the zebrafish (Danio rerio) pharyngeal dentition (Teleostei, Cyprinidae). Anat. Embryol. 198: 289-305.

HUYSSEUNE, A. and WITTEN, P.E. (2006). Developmental mechanisms underlying tooth patterning in continuously replacing osteichthyan dentitions. J. Exp. Zool. 306B: 204-215.

JACKMAN, W.R., DRAPER, B.D. and STOCK, D.W. (2004). Fgf signaling is required for zebrafish tooth development. Dev. Biol. 274: 139-157.

JAMORA, C., DASGUPTA, R., KOCIENIEWSKI, P. and FUCHS, E. (2003). Links between signal transduction, transcription and adhesion in epithelial bud development. Nature 422: 317-322.

JENSEN, B.L. and KREIBORG, S. (1990). Development of the dentition in cleidocranial dysplasia. J. Oral Pathol. Med. 19: 89-93.

LAURENTI, P., THAERON-ANTONO, C., ALLIZARD, F., HUYSSEUNE, A. and SIRE, J.-Y. (2004). The cellular expression of eve1 suggests its requirement for the differentiation of the ameloblasts and for the initiation and morphogenesis of the first tooth in the zebrafish (Danio rerio). Dev. Dynam. 230: 727-733.

MELFI, R.C. (1982). Permar's Oral Embryology and Microscopic Anatomy. Seventh Edition. Lea \& Febiger, Philadelphia.

MJÖR, I.A. and FEJERSKOV, O. (1979). Histology of the human tooth. 2nd Edition. Munksgaard, Copenhagen.

PETERKOVÁ, R., PETERKA, M., VIRIOT, L. and LESOT, H. (2000). Dentition development and budding morphogenesis. J. Craniofac. Genet. Dev. Biol. 20: 158-172.

PETERKOVÁ, R., PETERKA, M., VIRIOT, L. and LESOT, H. (2002). Development of the vestigial tooth primordia as part of mouse odontogenesis. Conn. Tiss. Res. 43: $120-128$.

REIF, W.E. (1982). Evolution of dermal skeleton and dentition in vertebrates: the odontode-regulation theory. Evol. Biol. 15: 287-368.

SIRE, J.-Y., DAVIT-BEAL, T., DELGADO, S., VAN DER HEYDEN, C. and HUYSSEUNE, A. (2002). First-generation teeth in nonmammalian lineages: Evidence for a conserved ancestral character? Microsc. Res. Techn. 59: 408434.

SMITH, M.M. (2003). Vertebrate dentitions at the origin of jaws: when and how pattern evolved. Evol. Dev. 5: 394-413.

TRAPANI, J. (2001). Position of developing replacement teeth in teleosts. Copeia 2001: 35-51.

VAN DER HEYDEN, C., ALLIZARD, F., SIRE, J.-Y. and HUYSSEUNE, A. (2005). 
Tooth development in vitro in two teleost fish, the cichlid Hemichromis bimaculatus and the cyprinid Danio rerio. Cell Tiss. Res. 321: 375-389.

VAN DER HEYDEN, C. and HUYSSEUNE, A. (2000). Dynamics of tooth formation and replacement in the zebrafish (Danio rerio) (Teleostei, Cyprinidae). Dev. Dynam. 219: 486-496.

VAN DER HEYDEN, C., HUYSSEUNE, A. and SIRE, J.-Y. (2000). Development and fine structure of pharyngeal replacement teeth in juvenile zebrafish (Danio rerio) (Teleostei, Cyprinidae). Cell Tiss. Res. 302: 205-219.

VAN DER HEYDEN, C., WAUTIER, K. and HUYSSEUNE, A. (2001). Tooth succession in the zebrafish (Danio rerio). Archs Oral Biol. 46: 1051-1058.

WAKE, D.B. (2003). Homology and Homoplasy. In Keywords \& Concepts in Evolutionary Developmental Biology. (Hall, B.K. \& Olsen, W.M., Eds.),Cambridge: Harvard University Press. pp. 191-201.

WANG, X.-P., ÅBERG, T., JAMES, M.J., LEVANON, D., GRONER, Y. and THESLEFF, I. (2005). Runx2 (Cbfa1) inhibits Shh signaling in the lower but not upper molars of mouse embryos and prevents the budding of putative successional teeth. J. Dent. Res. 84: 138-143.

WISTUBA, J., GREVEN, H. and CLEMEN, G. (2002). Development of larval and transformed teeth in Ambystoma mexicanum (Urodela, Amphibia): an ultrastructural study. Tissue Cel/34: 14-27.

WITTEN, P.E., HANSEN, A. and HALL, B.K. (2001). Features of mono- and multinucleated bone resorbing cells of the zebrafish Danio rerio and their contribution to skeletal development, remodeling and growth. J. Morphol. 250: 197-207.

Received: September 2005 Reviewed by Referees: October 2005 Modified by Authors and Accepted for Publication: April 2006 Published Online: July 2006 